\title{
An Enhanced LEACH Routing Algorithm for Energy Conservation in A Wireless Sensor Network
}

\author{
M. Saidu ${ }^{\mathrm{a}}$, E.N. Onwuka ${ }^{\mathrm{a}}$, M. Okwori ${ }^{\mathrm{a}}$, A. Umar ${ }^{\mathrm{a}}$ \\ Department of Telecommunications Engineering, Federal University of Technology Minna, Nigeria
}

\begin{abstract}
It has been well established that the lifetime of a Wireless Sensor Network (WSN) depends on the energy of the sensor nodes which is limited by the battery capacity of the nodes. This challenge has led to research efforts towards developing a more efficient energy based routing algorithms and architectures. One of such popular algorithm is the Low-Energy Adaptive Clustering Hierarchy (LEACH). The LEACH approach adopts randomized rotation of local base stations (or cluster-heads $(\mathrm{CH})$ ) to evenly distribute energy load amongst the sensors in the network. A variant of LEACH known as Vice-LEACH (V-LEACH) introduces the concept of a vice- $\mathrm{CH}$ that takes over the role of the main $\mathrm{CH}$ in the event of $\mathrm{CH}$ death. Random selection of cluster- head node in both LEACH and V-LEACH ignore node's residual energy and the optimal number and distribution of cluster head is not ensured in all rounds. This work presents an enhanced cluster-based routing algorithm which took into consideration the residual energy of nodes in the network while maintaining the optimal number of $\mathrm{CHs}$ throughout the network lifetime. A sensor network was developed on OPNET Modeler software tool and simulation tests were performed to evaluate the performance of the enhanced routing algorithm on the network. Simulation results showed that this enhanced routing algorithm distributed network energy consumption across the network nodes hence significantly improving the lifetime of the sensor network.
\end{abstract}

Index Terms: Wireless Sensor Network, Adaptive Clustering, Energy conservation, Sensor Network Lifetime

(C) 2016 Published by MECS Publisher. Selection and/or peer review under responsibility of the Research Association of Modern Education and Computer Science

\section{Introduction}

A wireless Sensor Network (WSN) usually comprises of a large number of battery operated micro- sensor nodes densely and randomly deployed over a geographical area to cooperatively monitor physical or environmental conditions at different locations (Ramesh \& Somasundaram, 2011). It is an infrastructure that comprises of sensing, computing, and communication elements (sensor nodes) that gives an end-user/controller

\footnotetext{
* Corresponding author. Tel.: 08039719789

E-mail address: samuslim@futminna.edu.ng
} 
the ability to observe and react to events and phenomena in a specified environment. WSN technology has attracted significant attention because of its large number of new applications in home automations, disasters and environmental monitoring, military operations, security surveillance, health services and other commercial purposes (Heinzelman, et.al 2000). The network has been described as an "exciting emerging domain of deeply networked system of lower-power wireless motes with a tiny amount of CPU and memory, and large federated networks for high-resolution sensing of the environment" (Kazem, .al, 2007). However, sensor nodes are resource constrained such as limited energy supply, low storage capacity, and weak computing ability. (Haosong \& Younghwan, 2010). It has been well established that the lifetime of a Wireless Sensor Network depends on the energy of the sensor nodes which is limited by the battery of the sensor nodes. This challenge has led to research efforts towards developing more efficient energy based routing algorithms and architectures. The main goal of routing algorithms is to set up energy-efficient paths between sensor nodes and the data sink such that the lifetime of the network is maximized.

Clustering in wireless sensor networks improves lifetime of the network by load distribution, data aggregation and use of cluster heads to efficiently route messages to the central gateway (R. Langer et. al. 2009). Several energy-efficient clustering routing algorithms for wireless sensor networks have been proposed in literature. Low-Energy Adaptive Clustering Hierarchy (LEACH) protocol is one of the earliest and a wellknown hierarchical routing protocol for WSN in literature. The LEACH approach adopts randomized rotation of local base- stations (or cluster-heads) to evenly distribute energy load among the sensors in the network. In this algorithm, the Cluster Head $(\mathrm{CH})$ is chosen randomly and in each round the role of $\mathrm{CH}$ is assigned to another node in order to balance the energy consumption in the whole network. Many clustering algorithms proposed in literature are improvements of the LEACH protocol and architecture. One of such improvements is the Vice-LEACH (V-LEACH) which introduced the concept of a Vice Cluster Head (V-CH) that takes over the role of the cluster head in the event of a cluster head death. Random selection of cluster head in both LEACH and V-LEACH ignore node's residual energy and the optimal number of cluster head is not constant in all rounds.

In this paper, an enhanced cluster-based energy-efficient routing algorithm is presented. Our algorithm selects the optimal number of cluster heads at network initialization, divides the network into optimal number of sectors, and then initializes the network with one node as a $\mathrm{CH}$ in each sector. At each round, each $\mathrm{CH}$ selects the node with highest residual energy in the cluster as a vice $\mathrm{CH}$. The $\mathrm{CH}$ turns the vice $\mathrm{CH}$ to sleep for that round and the vice $\mathrm{CH}$ becomes the $\mathrm{CH}$ in the next round. This algorithm considers the node's residual energy while maintaining the optimal number of $\mathrm{CHs}$ throughout the network lifetime.

This paper is presented in six (6) sections. Section two (2) presents a review of the hierarchical WSN routing algorithm with emphasis on the Low-Energy Adaptive Clustering Hierarchy (LEACH) algorithm. Section three (3) presents the description of the enhanced routing algorithm while the development of the algorithm on OPNET Modeler is presented in section four (4). Section five (5) presents and discusses the simulation results obtained and section six (6) concludes the work.

\section{Related Works}

The main goal of cluster-based (hierarchical-based) routing protocols is to improve energy efficiency of network sensor nodes and to increase the entire lifetime of the network (Amir, al., 2012). In clustering, the whole sensor network is divided into small regions known as clusters. In each cluster, one node is elected as cluster head. Each $\mathrm{CH}$ is responsible for aggregating the sensed data from its cluster member node(s) and propagates it to base station or to the next $\mathrm{CH}$. Clustering schemes offer reduced communication overheads, and efficient resource allocations thus decreasing the overall energy consumption and reducing the interferences among sensor nodes (V. Kumar et. al 2011). Low-Energy Adaptive Clustering Hierarchy (LEACH) is the first hierarchical cluster-based routing protocol for WSN which partitions the nodes into clusters (Heinzelman. et. al.. 2000). LEACH is a distributed algorithm which periodically elects the cluster heads randomly. Each round consists of two phases: - a set-up phase and a steady-state phase. In the set-up 
phase, the clusters are constructed and in the steady-state phase the data is acquired and transferred. Once the steady-state phase is finished, the next iteration begins. Fig. 1 illustrates flowchart of this protocol. This algorithm saves energy due to data aggregation, fair cluster head selection process and as a result of distributed clusters and nodes that make their decisions autonomously without any central control. This was achieved by each node generating a random number between 0 and 1 . If the number is smaller than the threshold value $T(n)$ in equation 1 , the node becomes the cluster head in this round.

$$
T(n)=\left\{\begin{array}{cc}
\frac{p}{1-p\left(r \bmod \frac{1}{p}\right)}, & \forall n \in G \\
0, & \text { else }
\end{array}\right.
$$

$\begin{array}{ll}p & \text { the predetermined percentage of cluster heads (e.g., } \mathrm{p}=0.05) \\ r & \text { the current round; and } \\ G & \text { the set of nodes that have not been cluster heads in the last } 1 / \mathrm{P} \text { rounds. }\end{array}$

After $1 / \mathrm{p}$ rounds, the process start again and all nodes will be in the set $\mathrm{G}$ for cluster head selection mechanism. Each node that has elected itself as cluster head for the current round will broadcasts an advertisement message to the rest of the nodes in the network. All the non-cluster head nodes, after receiving this advertisement message, decide on the cluster to which they will belong for this round. Once the cluster heads are decided, the steady-state phase begins the time when sensor nodes start sensing and transmitting data to their cluster-heads. Another variant of LEACH algorithm is LEACH Centralized (LEACH-C) (Wendi B., al., 2002). This is identical to the LEACH protocol as far as formatting clusters at the beginning of each round. However, instead of nodes randomly self-selecting the $\mathrm{CH}$, a centralized algorithm is performed by the sink in LEACH-C. In this case, the sink collects location information from the nodes, decides the $\mathrm{CH}$ and then broadcasts its decision to the nodes.

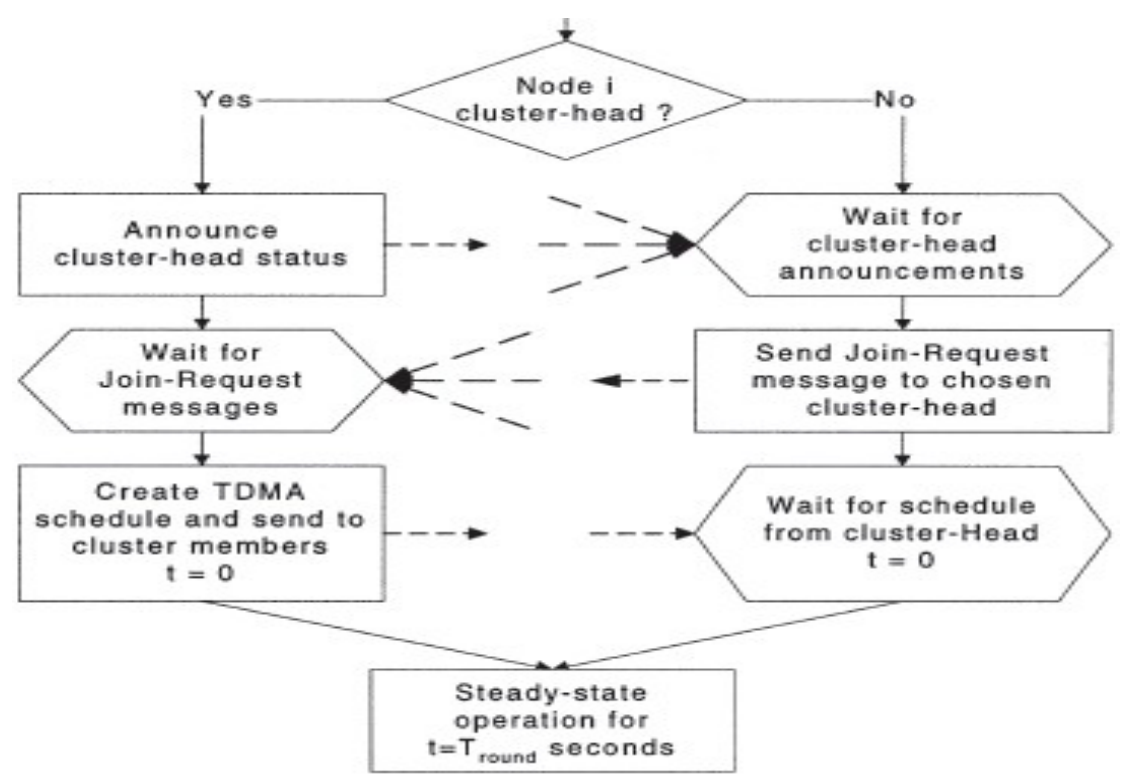

Fig.1.Flow chart of LEACH Algorithm (Wendi B., al., 2002) 
The overall performance of LEACH-C is better than LEACH since it moves the duty of cluster formation to the sink. However, LEACH-C is sensitive to the sink location. Once the energy cost of communicating with the sink becomes higher than the energy cost for cluster formation, LEACH-C no longer provides good performance. Dependence on the sink location is a major disadvantage of LEACH-C.

A technique known as Energy-LEACH (E-LEACH) protocol improves the $\mathrm{CH}$ selection procedure of LEACH by making the residual energy of nodes as the main metric that determines whether the nodes turn into $\mathrm{CH}$ or not after the first round (Fan \& Song, 2007). In the first iteration, nodes are randomly selected as $\mathrm{CH}$. Subsequently, nodes with more residual energy are selected as $\mathrm{CH}$ in the remaining round.

Another mechanism known as Multi-hop-LEACH protocol is presented in (Fan \& Song, 2007). It is almost the same as LEACH protocol, except that it makes communication mode from single hop to multi-hop between cluster heads and sink. That is, multi-hop communication is adopted among CHs. Based on the selected optimal path, these $\mathrm{CHs}$ transmit data to the corresponding $\mathrm{CH}$ nearest to the sink. Finally, this $\mathrm{CH}$ sends data to the sink.

LEACH's stochastic CH selection algorithm is extended in (Ray \& De, 2011) by adjusting the threshold $T(n)$ in equation 1. The threshold included; residual energy of the nodes, distance between the nodes and the base station and the number of consecutive rounds in which a node has not been a cluster head as metric for selecting cluster heads.

In (Anindita \& De, 2012), the LEACH protocol is enhanced by improving the election strategy of the cluster-head nodes. The enhanced protocol considered the residual energy, distance from base station, number of consecutive rounds in which a node has not been a $\mathrm{CH}$ and also the factor that whether the nodes remaining energy is sufficient enough to send the aggregate data to the base station or not.

Finally, another variant known as the Vice-LEACH approach introduces the concept of an assistant which resumes duty as full cluster head in the event of the main $\mathrm{CH}$ death (Bani. et. al 2009). However, it was observed that this approach failed to account for the optimal number of $\mathrm{CH}$ selection as a function of the algorithm's performance. This is the focus of our enhanced routing algorithm.

\section{Description of the Enhanced Routing Algorithm}

This algorithm intends to improve on certain limitation of the previous algorithms which includes the following:

- Sub-optimal CH selection process

- Inefficient energy conservation process.

The enhancement process is divided into the Network Initialization phase and Network Operation Phase. The solution to the problem of sub-optimal $\mathrm{CH}$ selection process was incorporated into the network initialization process. This was achieved by hard-coding the original $\mathrm{CH}$ candidates before Sensor nodes are deployed. To obtain an efficient realization, the sensing area was assumed identified and geometrically divided into $\mathrm{N}$ equally sized grids. Sensors were then carefully deployed into each grid box with an initialized CH node per batch. This introduces a simple solution to the challenge of optimal $\mathrm{CH}$ selection by ensuring that a single $\mathrm{CH}$ is deployed per grid. It was presumed that all sensing areas including irregularly shaped sensing areas are on rectangular coordinates (grids). This ensures a total of $\mathrm{N}$ initial $\mathrm{CHs}$ per total sensing area. Furthermore, designers can now ensure that only one $\mathrm{CH}$ is deployed per grid. The operation began with the initialization of the originally hard-coded CHs deployed in each grid of the sensing area along with other sensor nodes.

Various terms used in the description of the algorithm are defined in Table 1. 
Table 1. Definition of terms

\begin{tabular}{ll}
\hline Terms & Definitions \\
\hline$S i$ & Sensor Node $(\mathrm{i}=1,2,3,4,5)$ \\
$C H$ & Cluster-Head sensor node \\
$V C H$ & Vice-Cluster Head sensor node \\
$E i$ & Energy of Sensor Node $(\mathrm{i}=1,2,3,4,5)$ \\
$E s$ & Energy spent for sending data packet \\
$E r$ & Energy spent for receiving data packet \\
$E a s$ & Energy spent for aggregating and sending data packet by $\mathrm{CH}$ to the base station \\
$\mathrm{CH}_{a d-p k}$ & Cluster-Head advertise packet \\
$\mathrm{CH}_{b d-p k}$ & Cluster bind request packet \\
$V C H_{s t s-p k}$ & VCH status packet \\
$V C H_{a w k-p k}$ & VCH awake packet. \\
\hline
\end{tabular}

Fig 2 Represents the flowchart of the enhanced algorithm which follows the seven steps outlined below:

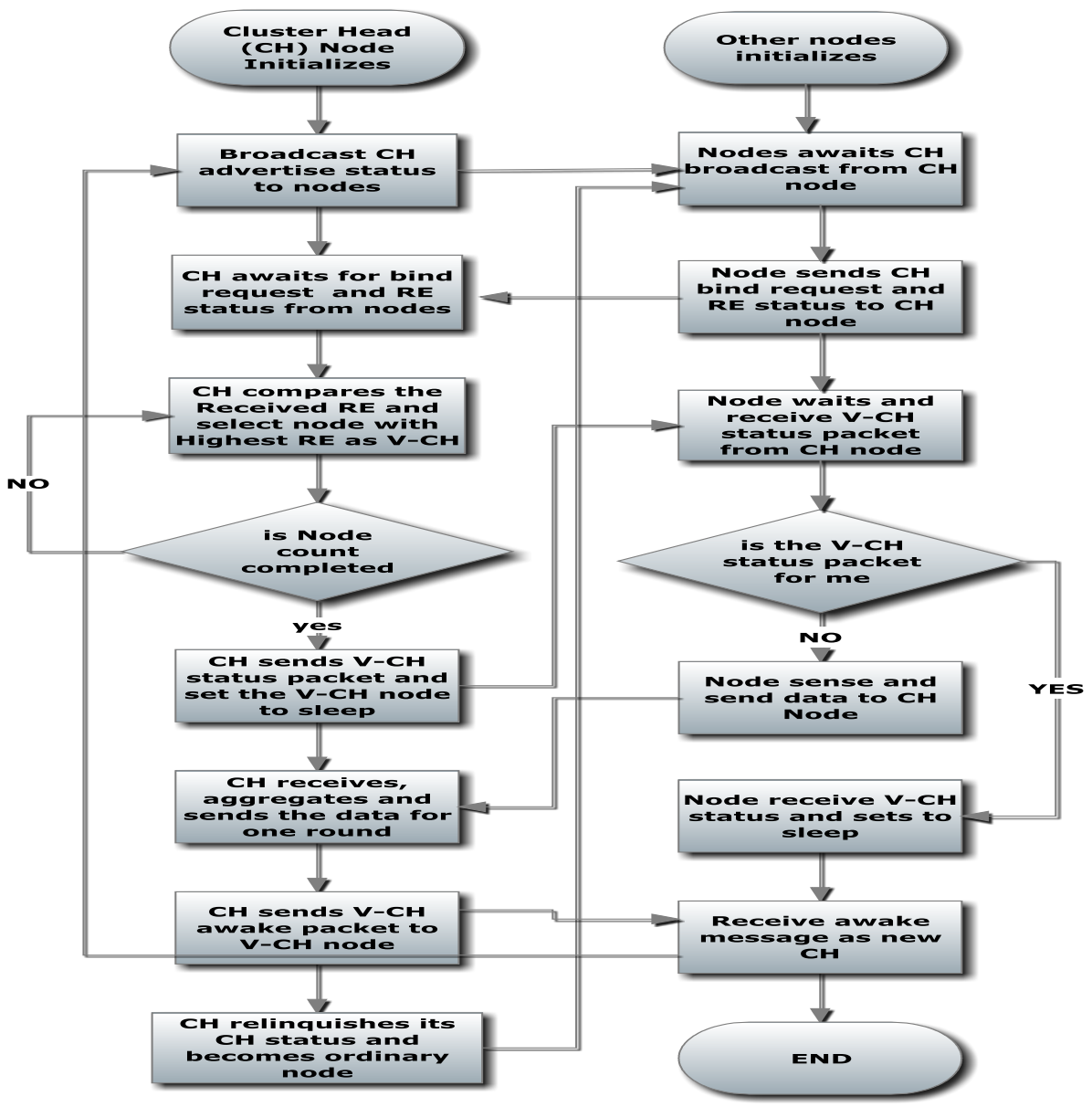

Fig.2.Flowchart of the Enhanced algorithm (Muslim, S. et. al 2013) 
Step 1: $\boldsymbol{C H}$ sends $\boldsymbol{C H} \boldsymbol{H}_{a d-p k}$ to $\boldsymbol{S i}$ in a network.

Step 2: Each $\boldsymbol{S i}$ sends $\boldsymbol{C} \boldsymbol{H}_{\boldsymbol{b d}-\mathrm{pk}}$ to $\boldsymbol{C H}$. The packet also contains $\boldsymbol{E} \boldsymbol{i}$

Step 3: $\boldsymbol{C H}$ compares the $\boldsymbol{E} \boldsymbol{i}$ and select $\boldsymbol{S i}$ with highest $\boldsymbol{E} \boldsymbol{i}$ as $\boldsymbol{V C H}$

Step 4: $\boldsymbol{C H}$ sends $\boldsymbol{V} \boldsymbol{C H} \boldsymbol{H}_{s t s-p k}$ to the $\boldsymbol{V C H}$. The received packet sets the $\boldsymbol{V C H}$ to sleep.

Step 5: Other $\boldsymbol{S i}$ senses and sends data packet to the $\boldsymbol{C H}$

Step 6: $\boldsymbol{C H}$ aggregates and sends the aggregated data to the base station.

Step 7: $\boldsymbol{C H}$ relinquishes its status and sends $\boldsymbol{V} \boldsymbol{C H} \boldsymbol{H}_{a w k-p k}$ to the $\boldsymbol{V C H}$ to take-over as a new $\boldsymbol{C H}$

Step 1 to re-run for next round of data transmission.

Table 2.0 shows the initial energies assigned to the sensor nodes ( $\mathrm{Si}$ ) and also the energy spent on different processes involved in our model.

Table 2.Energy Assignment

\begin{tabular}{lll}
\hline Definition & Symbol & Value $(\mathbf{J})$ \\
\hline Initial Energy of $S_{1}$ & $E_{1}$ & 2.0005 \\
Initial Energy of $S_{2}$ & $E_{2}$ & 2.0001 \\
Initial Energy of $S_{3}$ & $E_{3}$ & 2.0002 \\
Initial Energy of $S_{4}$ & $E_{4}$ & 2.0004 \\
Initial Energy of $S_{5}$ & $E_{5}$ & 2.0003 \\
Energy spent for sending data packet & $E s$ & 0.0100 \\
Energy spent for receiving data packet & $E r$ & 0.0010 \\
Energy spent for aggregate and sent data packet to the & & \\
base-station & Esa & 0.1000 \\
\hline
\end{tabular}

Our model was based on the following assumption:

1) The initial energy of sensor nodes ( $\mathrm{Si}$ ) differed by a little margin as listed in Table 2.0. This assumption was made to establish the differences in residual energy among sensor nodes and to support the fact that the battery of most devices usually have little discrepancy when measured with a very sensitive meter.

2) Nodes in a cluster consumes equal amount of energy for packet transmission and also for receiving data packet.

3) The lifetime of the network ends when the residual energy of a node in the network is nearly zero.

\section{Development of the Model Using OPNET Modeler}

The algorithm was model using OPNET Modeler 14.5. The OPNET design procedure follows a three tiered hierarchy that includes: The Network Project Model, the Node Model and the Process Model.

The Network Project Model represent the network topology which is described in terms of sub networks, nodes, links and geographical context. The Node Model specifies objects in network domain and the node's internal architecture in terms of functional elements and data flow between them. The functional elements are the processors, queues and transceivers. The processor are fully programmable via their process model, queues buffers and also manages data packets while transceivers are node interfaces. The interfaces between node modules can be packet stream or statistic wires. The Process Model describes the behavior of processes (algorithms, Protocols, applications) specified using Finite State Machines (FSM) and extended high-level language. The FSMs run on sets of programming codes referred to as PROTO-C, which combines C++ codes and indigenous OPNET codes (M. Okwori. et. al. 2014).

The Network Project Model designed for this work is shown in Figure 3.0. The model consist of one initial cluster head node $(\boldsymbol{C H})$ and four (4) other sensor node (Si). Node Model and Process Model were designed for each of the $\boldsymbol{C H}$ and $\boldsymbol{S i}$. The residual energy of each node as it was sending and receiving packets during network simulation were collected as a metric to measure the performance of the algorithm. 


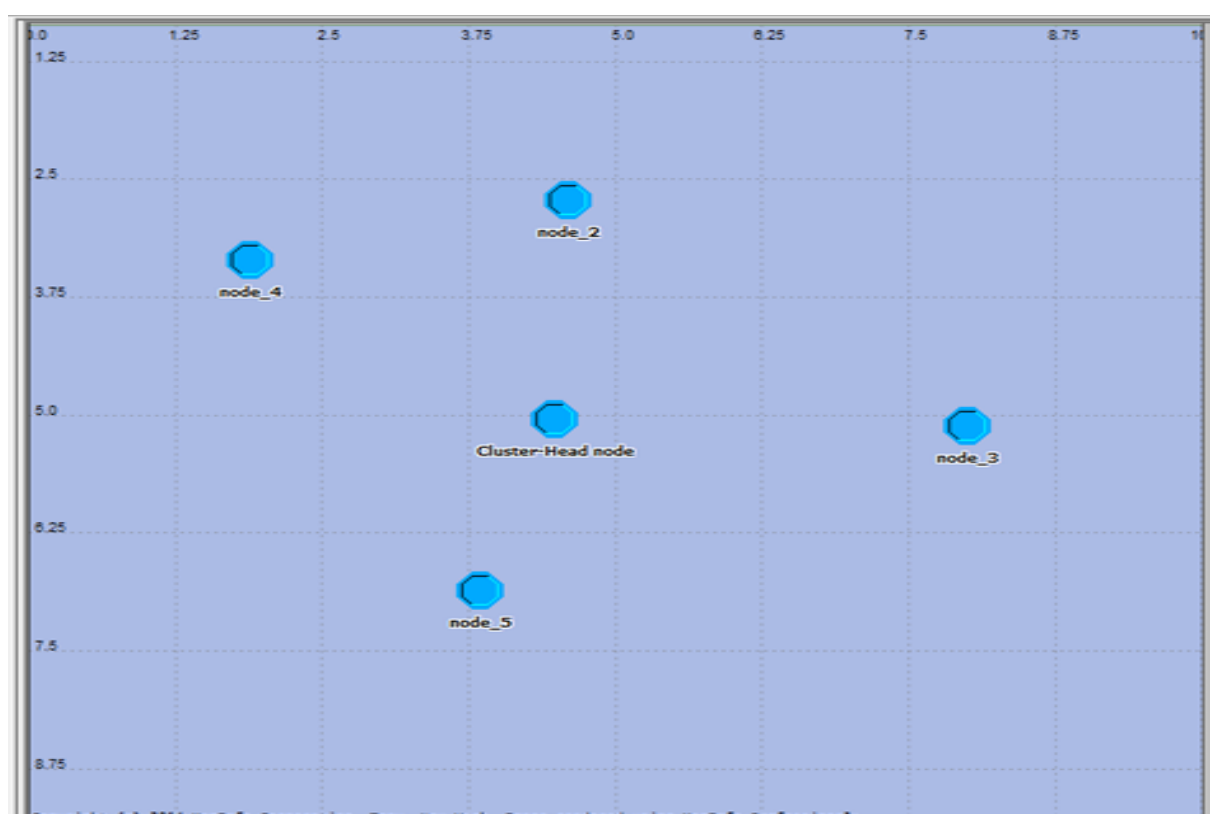

Fig.3.Network Project Model.

\section{Simulation Results and Discusion}

Figure 4.0 shows the graphs of Residual Energy level interaction against simulation time of five nodes deployed in the network project model shown in Figure 3.0. The initial energy of all nodes started at approximately $2 \mathrm{~J}$ and each decreases linearly to approximately zero during the simulation period. Each individual node's graph is characterised by; long vertical drop, short vertical drop, small slant drop and also maintaining a certain energy value at different simulation periods as depicted on the Figure.

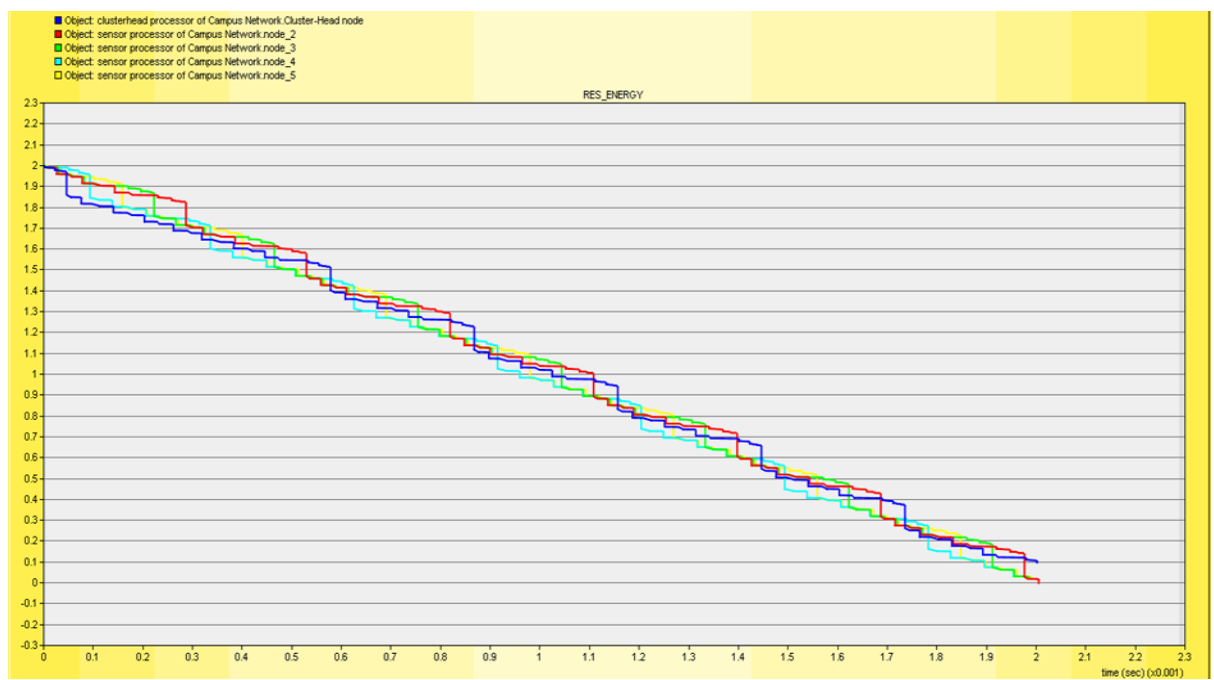

Fig.4.Residual Energy Level Interaction of Network Nodes. 
The long vertical drop in residual energy of a node is the energy loss $\left(\operatorname{Er}_{(i-1)}+E a s\right)$ when the node acted as a cluster head node. It losses energy $\left(E r_{(i-1)}\right.$ to receive data packets from other non $\mathrm{CH}$ nodes and (Eas) to send the aggregated data to the base station. The short vertical and small slant drops in residual energy of a node are the energy losses ( $E s$ and $E r$ ) when the non-cluster head node sent and received data packet to and from $\mathrm{CH}$ node respectively. However, the sleeping $\mathrm{VCH}$ node with the highest residual energy maintained a certain energy value before it became $\mathrm{CH}$ in the next round. Each operation round began when the $\mathrm{CH}$ broadcasts $\mathrm{CH}$ advertise packet and ended when it sent the aggregated received data to the base station. The long vertical line in the graph therefore, marked the end of a round. The initialized $\mathrm{CH}$ node was labelled with Blue colour while the other four nodes_2, 3, 4, and 5 were labelled respectively as depicted on top of the graph.

The Residual Energy (RE) level interaction of Figure 4.0 was reproduced in Table 2

The table showed: the initial energy of each node, the Residual Energy (RE) level of each node at the end of every round, the Cluster Head $(\mathrm{CH})$ node and the Vice Cluster-Head $(\mathrm{V}-\mathrm{CH})$ node at that particular round.

Table 3. Residual Energy (RE) Level Interaction of Network Nodes

\begin{tabular}{|c|c|c|c|c|c|c|c|}
\hline $\begin{array}{l}\text { Round } \\
\text { No }\end{array}$ & $\begin{array}{c}\text { Node } 1 \\
\text { RE }(J)\end{array}$ & Node 2 RE(J) & Node 3 RE(J) & Node 4 RE(J) & Node 5 RE(J) & $\begin{array}{c}\text { CH } \\
\text { Node }\end{array}$ & $\begin{array}{l}\text { V-CH } \\
\text { Node }\end{array}$ \\
\hline Initial & 2.0005 & 2.0001 & 2.0002 & 2.0004 & 2.0003 & Node 1 & \\
\hline 1 & 1.8675 & 1.9561 & 1.9562 & 1.9874 & 1.9563 & Node 1 & Node 4 \\
\hline 2 & 1.8145 & 1.9121 & 1.9122 & 1.8544 & 1.9433 & Node 4 & Node 5 \\
\hline 3 & 1.7715 & 1.8681 & 1.9892 & 1.8004 & 1.8004 & Node 5 & Node 3 \\
\hline 4 & 1.7285 & 1.8551 & 1.7662 & 1.7564 & 1.7563 & Node 3 & Node 2 \\
\hline 5 & 1.6855 & 1.7221 & 1.7123 & 1.7434 & 1.7123 & Node 2 & Node 4 \\
\hline 6 & 1.6425 & 1.6881 & 1.6682 & 1.6104 & 1.6993 & Node 4 & Node 5 \\
\hline 7 & 1.5995 & 1.6241 & 1.6552 & 1.5564 & 1.5663 & Node 5 & Node 3 \\
\hline 8 & 1.5565 & 1.6111 & 1.5222 & 1.5124 & 1.5123 & Node 3 & Node 2 \\
\hline 9 & 1.5435 & 1.4781 & 1.4782 & 1.4781 & 1.4683 & Node 2 & Node 1 \\
\hline 10 & 1.4105 & 1.4241 & 1.4141 & 1.4554 & 1.4242 & Node 1 & Node 4 \\
\hline 11 & 1.3575 & 1.3801 & 1.3802 & 1.3224 & 1.4113 & Node 4 & Node 5 \\
\hline 12 & 1.3145 & 1.3361 & 1.3672 & 1.2684 & 1.2783 & Node 5 & Node 3 \\
\hline 13 & 1.2715 & 1.3231 & 1.2343 & 1.2244 & 1.2243 & Node 3 & Node 2 \\
\hline 14 & 1.2585 & 1.1901 & 1.1802 & 1.1802 & 1.1803 & Node 2 & Node 1 \\
\hline 15 & 1.1255 & 1.1361 & 1.1362 & 1.1674 & 1.1363 & Node 1 & Node 4 \\
\hline 16 & 1.0344 & 1.0921 & 1.0922 & 1.0344 & 1.1233 & Node 4 & Node 5 \\
\hline 17 & 1.0295 & 1.0481 & 1.0792 & 0.9804 & 0.9903 & Node 5 & Node 3 \\
\hline 18 & 0.9865 & 1.0351 & 0.9462 & 0.9364 & 0.9363 & Node 3 & Node 2 \\
\hline 19 & 0.9735 & 0.9021 & 0.8922 & 0.8923 & 0.8923 & Node 2 & Node 1 \\
\hline 20 & 0.8405 & 0.8481 & 0.8482 & 0.8794 & 0.8483 & Node 1 & Node 4 \\
\hline 21 & 0.7875 & 0.8041 & 0.8041 & 0.7464 & 0.8353 & Node 4 & Node 5 \\
\hline 22 & 0.7445 & 0.7601 & 0.7912 & 0.6924 & 0.7023 & Node 5 & Node 3 \\
\hline 23 & 0.7025 & 0.7471 & 0.6582 & 0.6484 & 0.6483 & Node 3 & Node 2 \\
\hline -- & -- & -- & -- & -- & -- & -- & -- \\
\hline-- & -- & -- & -- & -- & -- & -- & -- \\
\hline 35 & 0.1185 & 0.0281 & 0.0282 & 0.0282 & 0.0283 & Node 2 & Node 1 \\
\hline
\end{tabular}


The graphs of Figure 4.1 as well as Table 2 revealed the following facts:

- Node_1 (blue colour) was designed to initialize the network as Cluster-Head node while the other four nodes where designed to initialize the network as non-cluster head node (ordinary nodes).

- Node_1, as $\mathrm{CH}$ node in round one lost more energy for receiving and sending data packet from and to other nodes and also for aggregating and sending data to the base station while the other nodes lost less energy for just sending and receiving packets to and from $\mathrm{CH}$ node. Node_4, the V-CH node, had the highest residual energy after the first round and therefore became the $\mathrm{CH}$ node in the second round.

- This pattern of $\mathrm{CH}$ selection process continued as depicted in Figure 4 and as well in Table 3.

- The network lifetime ended after thirty five (35) rounds of operation and each node acted as cluster head for seven (7) rounds. This was to ensure the evenly distribution of energy consumption load across the network nodes.

- The residual energy of all the nodes finished almost at the same time due to the even distribution of network energy load across the network nodes.

We also investigate the performance of the algorithm when the initial energy of each node is doubled (4J) and the result obtained in this scenario is shown in Figure 5.0.

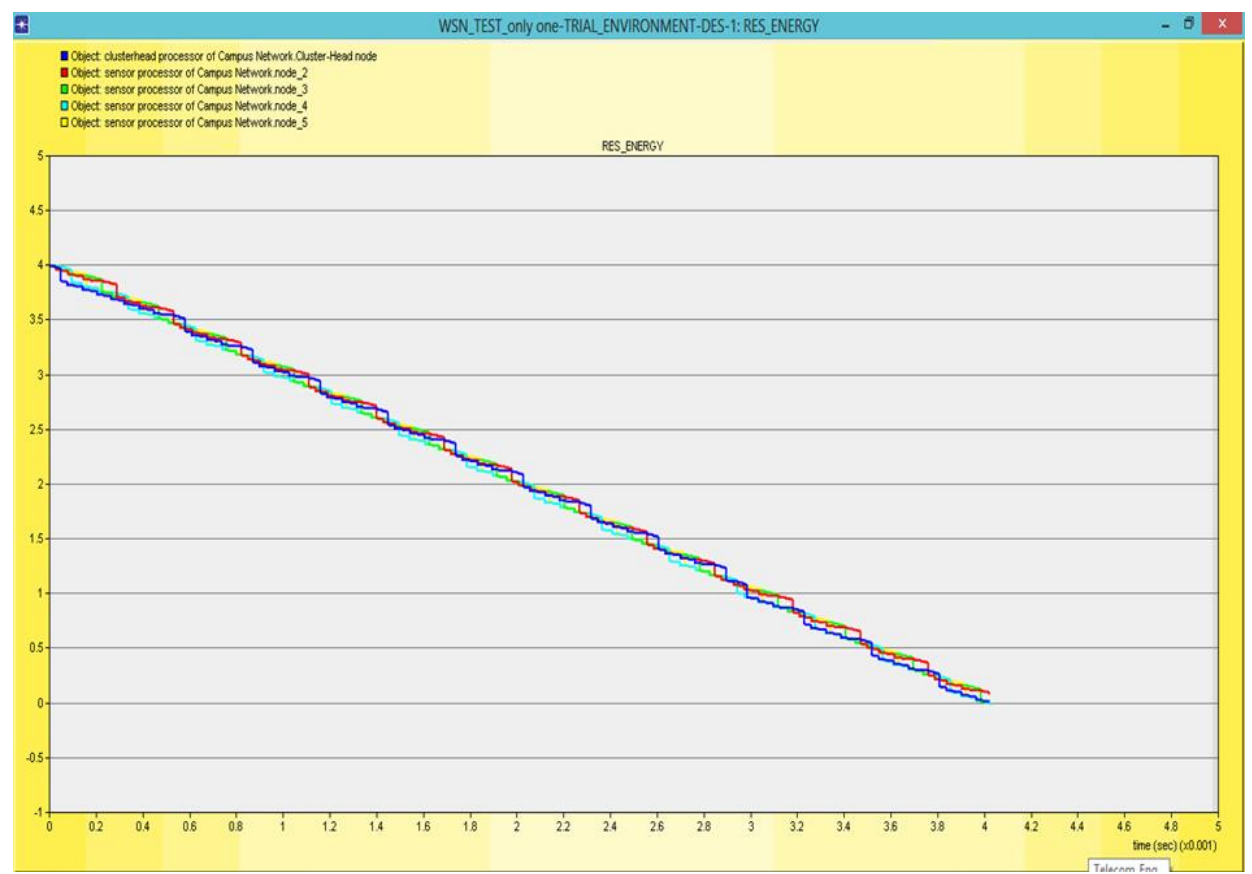

Fig.5.Residual Energy Levels Interaction of Network Nodes at 4J

The algorithm maintained the same pattern of $\mathrm{CH}$ selection process, the number of times each node acted as a $\mathrm{CH}$ node doubled and consequently the network lifetime doubled. The network lifetime ended after seventy (70) rounds of operation and each node acted as cluster head for fourteen (14) rounds

We also implemented the technique of random selection (Random-Based) of cluster head node adopted by LEACH and V-LEACH in this platform to evaluate the performance of our algorithm. We used different seed values in the OPNET code to acquire different randomness and the result obtained is shown in Figure 6.0. 


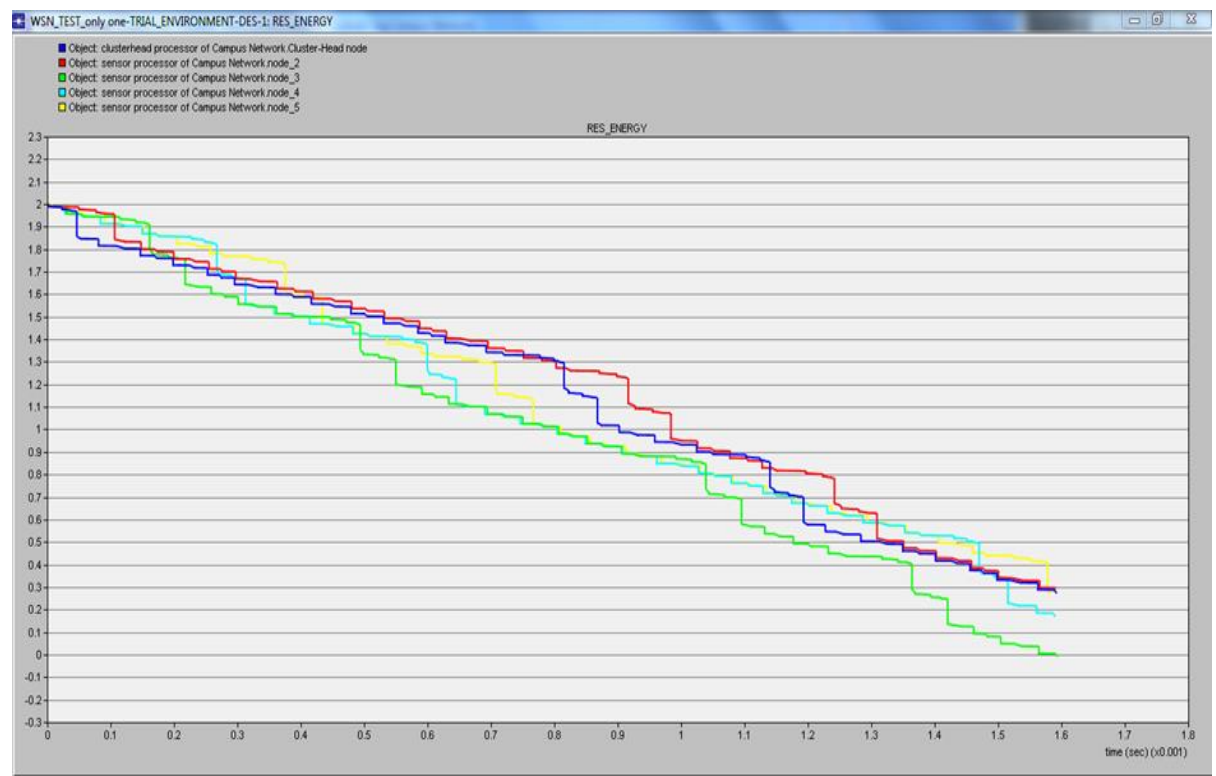

Fig.6.Residual Energy Level Interaction of Network Nodes (Random Selection Technique)

From Figure 6: there is more discrepancies in the residual energy of the network nodes due to uneven distribution of network energy consumption load across the network nodes in the random-based techniques. Table 4 compared the performance of our algorithm (Energy-Based Technique) and that of Random-Based Technique. The Table compared the residual energy level of network nodes of Figure 4/Table 3 and that of Figure 6 at the end of network lifetime.

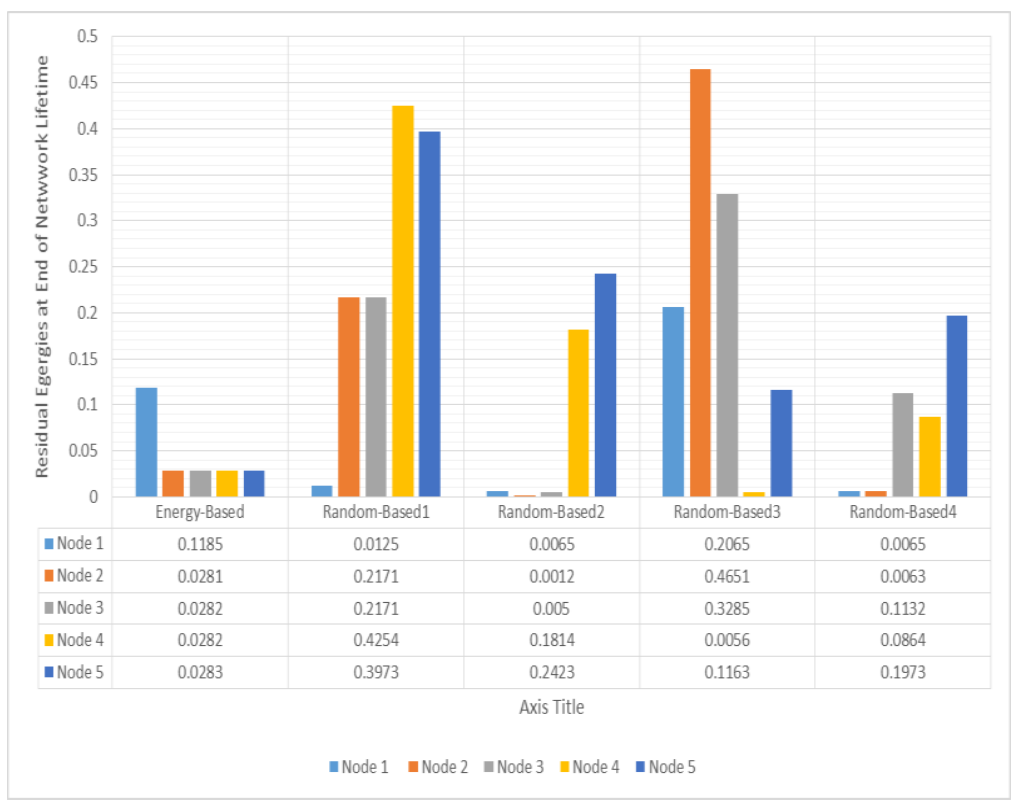

Fig.7.Comparison of Residual Energy (RE) Level Interaction of Network Nodes 
From Figure 7, it can be clearly seen that there is more discrepancies in the residual energy of nodes in the random-based technique due to uneven distribution of network energy consumption load across the network nodes. Consequently, the lifetime of the network decreased by $18.263 \%$ (from 200145(s) to average 163593(s)) and the number of rounds of operation attained also decreased by $18.571 \%$ (from 35 to average 29)

\section{Conclusions}

The challenge of efficient use of energy resources to prolong the lifetime of sensor network was identified and energy conservation has remained the key desire of any WSN routing algorithm. This work designed an enhanced Routing Algorithm to address the problems of random selection of $\mathrm{CH}$ node and sub-optimal cluster head selection process identified in the previous works. The algorithm took into consideration the residual energy of nodes in the network while maintaining the optimal number of $\mathrm{CHs}$ throughout the network lifetime. The algorithm was developed using OPNET modeler software tool. Simulation results obtained showed that our proposed algorithm achieved efficient network energy load distribution across the network nodes. The performance of the algorithm (Energy-Based Technique) was compared with the Random-Based Technique and the results showed an $18.26 \%$ dropped in the lifetime of the network and more discrepancies in the residual energy of the network nodes due to the uneven distribution of network energy consumption load across the network nodes in the random-based techniques. Our proposed algorithm distributes network energy consumption across all the network nodes hence improved the lifetime of A Wireless Sensor Network.

\section{References}

[1] Ramesh, \& Somasundaram. (2011). "A Comparative Study Of Clusterhead Selection Algorithms in Wireless Sensor Networks" International Journal of Computer Science \& Engineering Survey(IJCSES) Vol. 2, No 4,.

[2] Heinzelman, W., A.Chandrakasan, \& Balakrishman, H. (2000). "Energy-Efficient Communication Protocol For Wireless Microsensor Networks" Proceedings of the 33rd Hawaii International Conference on System Sciences.

[3] Kazem, S., Daniel, M., \& Znati, T. (2007). "WIreless Sensor Networks. Technology, Protocols, and Applications". A JOHN WILEY \& SONS, INC, PUBLICATION.

[4] Haosong, G., \& Younghwan, Y. (2010). "An Energy Balancing LEACH Algorithm for Wireless Sensor Networks" Seventh International Conference on Information Technology (pp. 822-827). IEEE Computer Society.

[5] Amir, K. A., Kamal, J., \& Mohammad, R. k. (2012). "Adaptive Clustering in Wireless Sensor Network": Considering Nodes With Lowest Energy. International Journal of Ad hoc, Sensor \& Ubiquitous Computing (IJASUC) Vol.3, April 2012.

[6] Wendi B., H., Chandrakasan, P., \& Hari, B. (2002). "An Appliation-Specific Protocol Architecture for Wireless Microsensor Networks" IEEE Transactions on Wireless Communications, Vol. 1, No 4, October 2002, (pp. 660-670)

[7] Fan, X., \& Song, Y. (2007). "Improvement on LEACH Protocol of Wireless Sensor Network". 2007 International Conference on Sensor Technologies and Applications (pp. 260-264). IEEE Computer Society.

[8] Ray, A., \& De, D. (2011). "Energy Efficient Clustering Hierarchy Protocol for Wireless Sensor Network" Proceeding of IEEE International Conference on Communication and Industrial Application(ICCIA), (pp. $1-4)$.

[9] Anindita, R., \& De, D. (2012). "Parametric Energy Cluster Head Selection Protocol for Wireless Sensor Network" international Journal of Advanced Computer Engineering \& Architecture Vol. 2, No 2 (JuneDecember, 2012), 239-245. 
[10] Bani, M., Alzou'bi, \& Khamayseh, Y. (2009). "Improvement on LEACH Protoccol of Wireless Sensor Network". International Journal of Digital Content Technology and its Applications, Volume 3, No 2, June 2009, 132-136.

[11] S. Muslim, O. Ugweje, E. N. Onwuka, and A. M. Aibinu. (2013) "An Enhanced Cluster Based Routing Algorithm for Energy Conservation in a Wireless Sensor Networks" 3rd Biennial Engineering Conference, Federal University of Technology Minna, May 2013

[12] M. Okwori, E.N. Onwuka, A.M. Aibinu. O.C. Ugweja. (2014) "Impelementation of a locator-Based Route Switching Scheme for Improved Routing in Proxy Mobile IPv6." International Journal of Wireless and Microwave Technologies, 2014, 4, 1-19.

[13] R. Langar, N. Bouabdallah, and R. Boutaba, "Mobility-aware clustering algorithms with interference constr aints in wireless mesh networks," Computer Networks, vol. 53, no. 1, pp. 25-44, 2009.

[14] V. Kumar, S. Jain, S. Tiwari. "Energy Efficient Clustering Algorithm in Wireless Sensor Network: A Survay" International Journal of Computer Sience Issues, Vol 8, issue 5, No 2, September 2011.

[15] A. Abbasi and M. Younis, "A survey on clustering algorithms for wireless sensor networks", Elsevier SCi, J. Computer Communications, Vo1.30, 2007, pp. 2826-2841.

\section{Authors' Profiles}
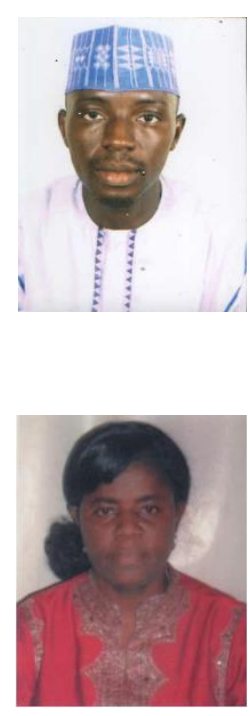

Saidu Muslim obtained a Bachelor of Engineering (B.Eng.) Degree from Electrical and Computer Engineering Department, Federal University of Technology (FUT) Minna, Niger State, Nigeria, in December, 2008 and a Master of Engineering (M.Eng.) Degree, in Telecommunication Engineering from Telecommunication Engineering Department, FUT, Minna, Niger State, Nigeria, in June 2014. He is currently an Assistant Lecturer in the Department of Telecommunication Engineering, Federal University of Technology, Minna, Nigeria. His Research interests includes Wireless Communication and Energy Resource Management in Wireless Sensor Network.

Elizabeth N. Onwuka obtained a Bachelor of Engineering (B.Eng.) Degree from Electrical and Computer Engineering Department, Federal University of Technology (FUT) Minna, Niger State, Nigeria, in October 1992; a Master of Engineering (M.Eng.) Degree, in Telecommunications, from Electrical and Computer Engineering Department, FUT, Minna, Niger State, Nigeria, in March 1998; and Doctor of Philosophy (PhD) Degree, in Communications and Information Systems Engineering, from Tsinghua University, Beijing, People's Republic of China, in June 2004. She is currently a professor in the Department of Telecommunications Engineering FUT, Minna, Niger State. Her research interest includes Mobile communications network architecture, IP networks, handoff management, paging, network integration, and resource management in wireless networks, wireless sensor networks.

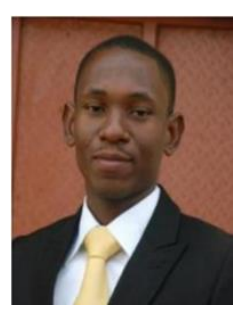

Michael Okwori is currently an Assistant Lecturer at the Department of Telecommunications Engineering, Federal University of Technology, Minna, Nigeria where he obtained his Masters of Engineering Degree in Communications Engineering in 2014. He obtained a First Class Honors Bachelor of Engineering Degree from the Department of Electrical and Computer Engineering, Federal University of Technology Minna in 2007. His Research interests include Mobility Management in IP Network, Mobile Telecommunication Architecture, Optical Communications and Wireless Sensor Networks. 


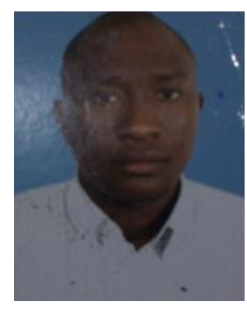

Abdullahi Umar obtained a Bachelor of Engineering (B.Eng.) Degree from Electrical and Computer Engineering Department, Federal University of Technology (FUT) Minna, Niger State, Nigeria, in December, 2008 and a Master of Engineering (M.Eng) Degree, in Telecommunication Engineering from Telecommunication Engineering Department, FUT, Minna, Niger State, Nigeria, in May 2014. He is currently an Assistant Lecturer in the Department of Telecommunication Engineering, Federal University of Technology, Minna, Nigeria. His Research interests include Radio Resource Management in WiMAX, Energy Management in Wireless Sensor Networks.

How to cite this paper: M. Saidu, E.N. Onwuka, M. Okwori, A. Umar,"An Enhanced LEACH Routing Algorithm for Energy Conservation in A Wireless Sensor Network",International Journal of Wireless and Microwave Technologies(IJWMT), Vol.6, No.4, pp.59-71, 2016.DOI: 10.5815/ijwmt.2016.04.06 